\title{
Endoscopic Management of Malignant Colonic Obstruction
}

\author{
Seung Young Seo and Sang Wook Kim \\ Division of Gastroenterology, Department of Internal Medicine, Biomedical Research Institute, Chonbuk National University Hospital and \\ Medical School, Jeonju, Korea
}

\begin{abstract}
Advanced colorectal cancer can cause acute colonic obstruction, which is a life-threatening condition that requires emergency bowel decompression. Malignant colonic obstruction has traditionally been treated using emergency surgery, including primary resection or stoma formation. However, relatively high rates of complications, such as anastomosis site leakage, have been considered as major concerns for emergency surgery. Endoscopic management of malignant colonic obstruction using a self-expandable metal stent (SEMS) was introduced 20 years ago and it has been used as a first-line palliative treatment. However, endoscopic treatment of malignant colonic obstruction using SEMSs as a bridge to surgery remains controversial owing to short-term complications and longterm oncological outcomes. In this review, the current status of and recommendations for endoscopic management using SEMSs for malignant colonic obstruction will be discussed. Clin Endosc 2020;53:9-17
\end{abstract}

Key Words: Colorectal cancer; Malignant colonic obstruction; Self-expandable metal stent

\section{INTRODUCTION}

Malignant colonic obstruction can be caused by colorectal cancer, metastatic cancer, and locally advanced pelvic tumors. Among these conditions, colorectal cancer is one of the most well-known and commonly diagnosed cancers worldwide. Up to $20 \%$ of patients with advanced colorectal cancer present with malignant colonic obstruction. ${ }^{1-5}$ Advanced colorectal cancer can cause acute colonic obstruction, which is a life-threatening condition that requires emergency decompression. Traditionally, malignant colonic obstruction has been treated using emergency surgery, including primary resection or stoma formation. However, owing to the relatively high rates of complications, such as anastomosis site

Received: February 18, 2019 Revised: July 18, 2019

Accepted: September 4, 2019

Correspondence: Sang Wook Kim

Division of Gastroenterology, Department of Internal Medicine, Biomedical Research Institute, Chonbuk National University Hospital and Medical School, 20 Geonji-ro, Deokjin-gu, Jeonju 54907, Korea

Tel: + 82-63-250-2302, Fax: + 82-63-254-1609, E-mail: clickm@jbnu.ac.kr ORCID: http://orcid.org/0000-0001-8209-540X

cc This is an Open Access article distributed under the terms of the Creative Commons Attribution Non-Commercial License (http://creativecommons.org/ licenses/by-nc/3.0) which permits unrestricted non-commercial use, distribution, and reproduction in any medium, provided the original work is properly cited. leakage, there is a need for alternative treatment strategies aside from emergency surgery. ${ }^{6-9}$ Endoscopic management of malignant colonic obstruction using a self-expandable metal stent (SEMS) was introduced approximately 20 years ago, and currently, its use as a palliative tool has yielded sufficient evidence for it to be accepted as a first-line treatment. However, SEMS placement, as a bridge to surgery (BTS), in patients with malignant colonic obstruction, which is potentially curable, remains controversial owing to concerns, such as shortterm complications and long-term oncological outcomes. In this review, the current status of and recommendations for endoscopic management using SEMSs for malignant colonic obstruction will be discussed.

\section{MATERIALS AND METHODS}

Since the 1990s, many types of colorectal stents have been developed. The majority of stents used for colonic obstruction are SEMSs; these are radiopaque, tube-shaped, metallic meshes, which are self-expandable. Once deployed, the stents expand for 2-3 days and are anchored to a designated site by a self-expandable force opposite to the lumen. SEMS materials can vary and can include the following: stainless steel, elgiloy, 
and nitinol. For a successful stent insertion, an endoscopist should be aware of the characteristics of each stent. Stainless steel stents are relatively stiff and interfere with magnetic resonance images (MRIs). Elgiloy stents are composed of cobalt, chromium, and nickel and have improved elasticity and flexibility compared with stainless steel stents; further, they do not affect MRI assessment. Nitinol stents are composed of nickel-titanium and yield a poor fluoroscopic visualization compared with elgiloy stents. To overcome this limitation, a radiopaque marker, such as a gold or silver marker, is added to both ends of these stents. These stents have more flexibility and can better hold the original shape than stainless steel and elgiloy stents and do not impact MRI assessments; therefore, nitinol stents are used worldwide.

Owing to the lack of research, it is not known whether an enema should be administered before colonic stent placement. Symptomatic colonic obstruction is a known contraindication for an enema; however, an enema can facilitate stent insertion by allowing for clear visualization. Prophylactic antibiotics are not recommended owing to the low incidence of reported bacteremia. Chun et al. reported that $6.3 \%(4 / 63)$ of patients showed positive blood culture test findings after colonic stent placement, and none of them developed infection symptoms within 48 hours. ${ }^{10}$ The prolonged procedure time, which was longer than 36 minutes, was the factor associated with bacteremia. ${ }^{10}$

Colonic stent insertion can be performed via either an endoscopic or a fluoroscopic method. Most colonic stents are inserted through endoscopy, followed by fluoroscopic guidance. Several studies have shown comparable technical success rates between endoscopic and fluoroscopic methods; however, a combination of both methods showed higher technical success rates. ${ }^{11-14}$ Therefore, the European Society of Gastrointestinal Endoscopy (ESGE) and the American Society of Gastrointestinal Endoscopy guidelines for malignant colonic obstruction recommend a combined approach of endoscopy and fluoroscopy. An appropriate learning curve should be anticipated for successful colonic stent placement. Two non-comparative studies suggested that at least $20 \mathrm{co}-$ lonic stents should be placed to increase the technical success rate and decrease the number of used stents. ${ }^{15,16}$ One retrospective study reported that endoscopists with experience in therapeutic endoscopic retrograde cholangiopancreatography (ERCP) achieved lower perforation rates during colonic stent placement. ${ }^{17}$ This demonstrates that a therapeutic ERCP endoscopist could have successfully mastered necessary techniques for performing colonic stents. To reduce complications, such as stent migration, a larger body diameter $(\geq 24 \mathrm{~mm})$ and a length of at least $2 \mathrm{~cm}$ on each side of the lesion are recommended. ${ }^{17-21}$ The overall process of colonic stenting for malignant obstruction is usually followed in this manner. The procedure does not need conscious sedation or general anesthesia. However, sedation with midazolam and analgesia is desirable in patients with excessive anxiety. After insertion of the colonoscope to the distal part of luminal obstruction with the patients in a supine or lateral decubitus position, a catheter loaded with a hydrophilic tip guidewire is inserted through the working channel to the distal part of the obstructive lesion. The catheter should be placed in the orifice of the obstructive lesion and parallelized with the bowel direction as far as possible to facilitate traversion of the obstructive lesion with the guidewire (Fig. 1A, B). Thereafter, cannulation with the guidewire is attempted gently. Endoscopists should be cautious of possible bleeding upon contact, which could be induced by too many cannulation trials or colonic perforation with the guidewire and perforation associated with excessive air inflation during this step. ${ }^{21}$ After traversing the obstructive lesion with the guidewire, the correct position of the guidewire should be confirmed via the fluoroscopic view. The catheter is then inserted to the proximal part of the obstructive le-
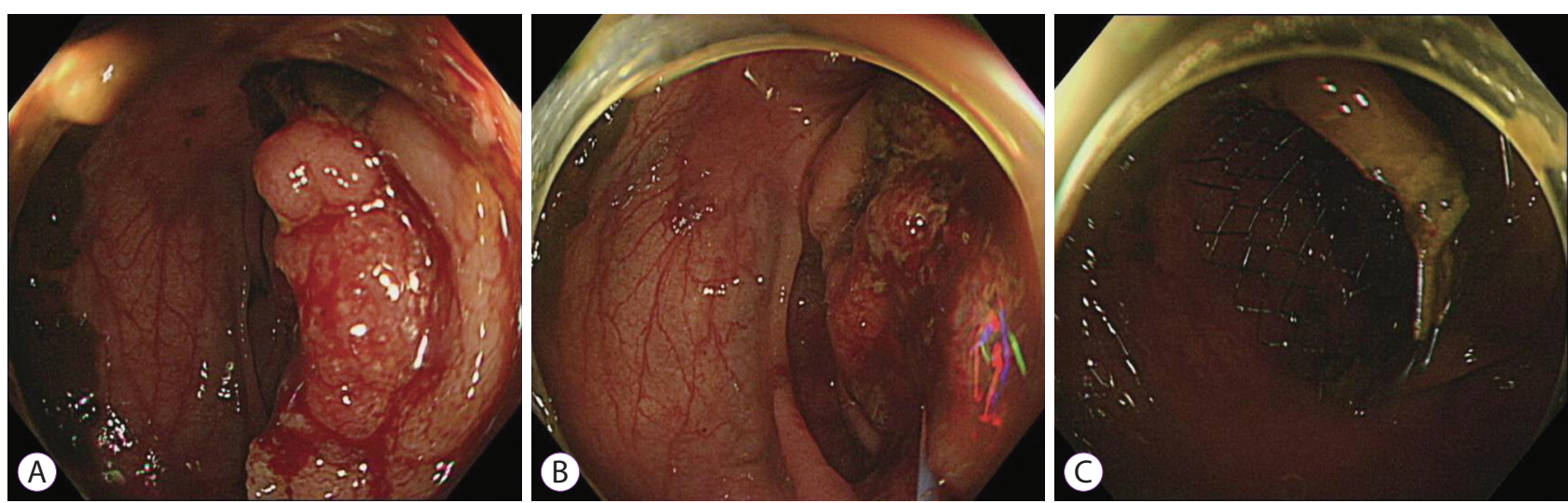

Fig. 1. Endoscopic images of colonic stenting. (A) Malignant obstruction at the splenic flexure, (B) cannulation, and (C) after deployment of the stent. 
sion over the guidewire, and a water-soluble contrast medium is injected to identify the length of the obstruction and to rule out the possibility of bowel perforation (Fig. 2A). The catheter is removed, leaving the guidewire tip as far as possible in the colon. A SEMS is inserted over the guidewire, and stent deployment is performed under endoscopic and fluoroscopic guidance (Figs. 1C, 2B). A stent length of at least $2 \mathrm{~cm}$ on each side of the lesion is required to prevent stent migration. After stent placement, a water-soluble contrast medium can be in- jected through the working channel to the stent for patency documentation and correct positioning of the stent, although it is not essential (Fig. 2C). Abdominal X-ray follow-up is required to verify bowel decompression and correct positioning of the stent (Fig. 3).

\section{Covered and uncovered stents}

Colorectal stents can be divided into two types: covered and
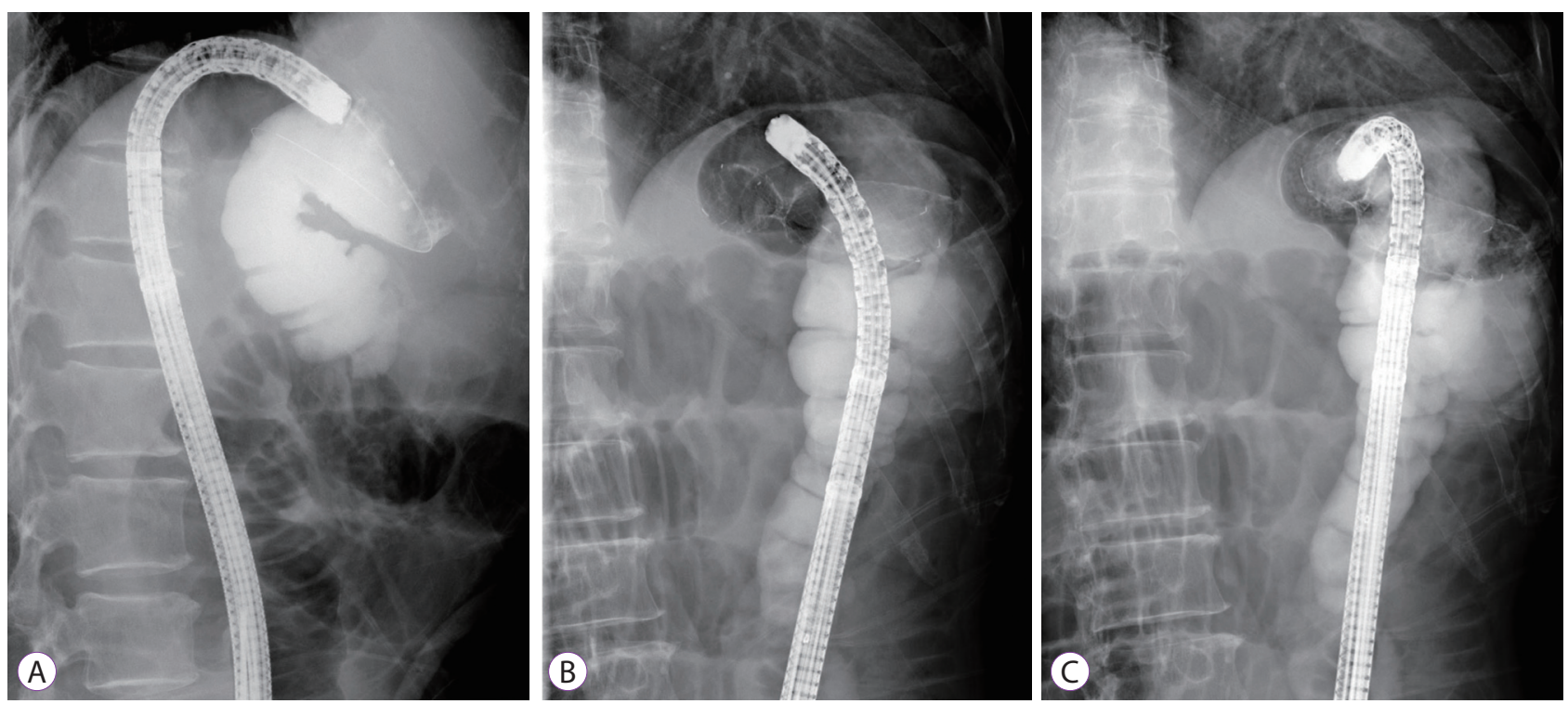

Fig. 2. Fluoroscopic images of colonic stenting. (A) Contrast medium injection after cannulation, (B) after deployment of the stent, and (C) documentation of stent patency and correct positioning.
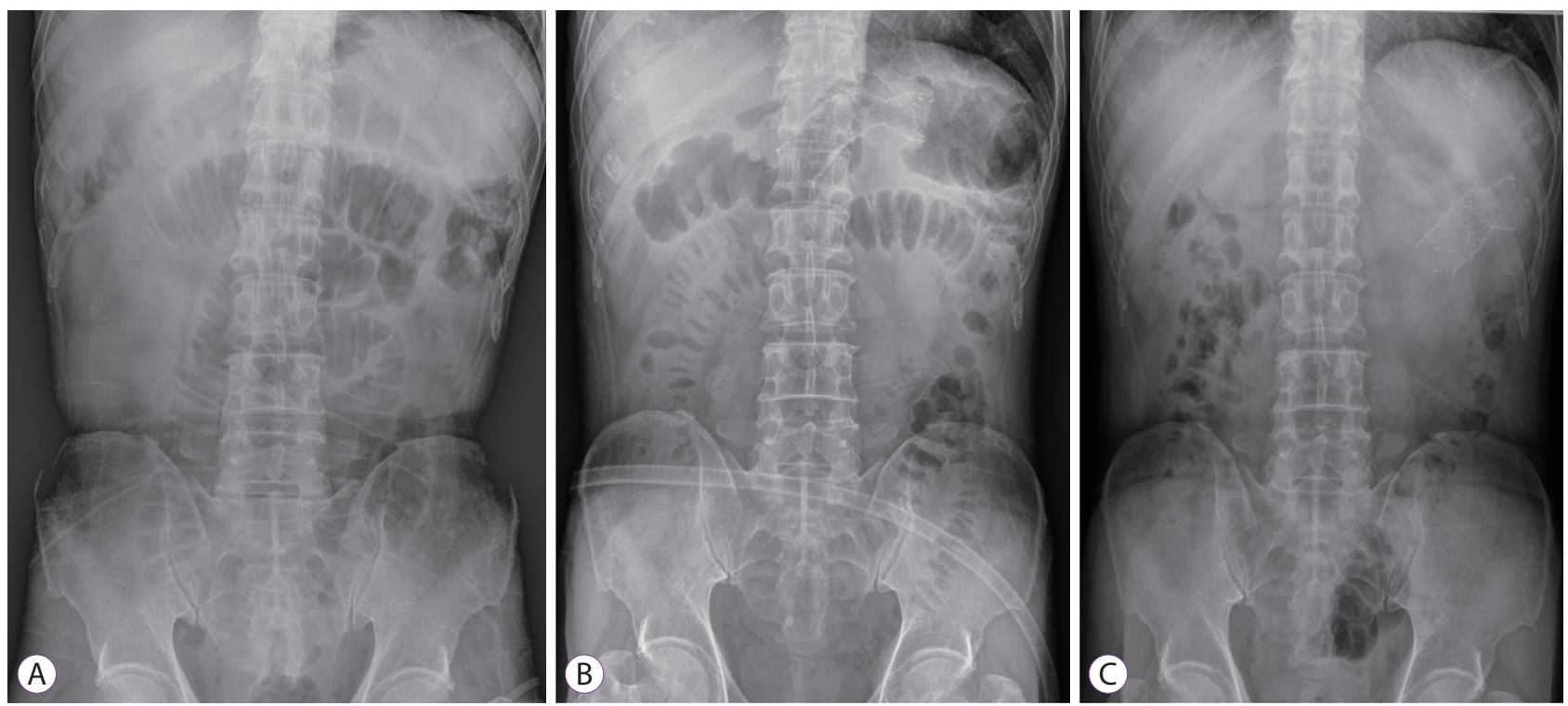

Fig. 3. Abdominal X-ray images showing bowel decompression and correct positioning of the stent. (A) Pre-colonic stenting, (B) 2 days after colonic stenting, and (C) 5 days after colonic stenting. 
uncovered. A covered stent is subdivided into two groupsfully and partially covered-and each type of this stent has specific advantages and disadvantages. Covered stents have a lower tumor ingrowth into the stent, which can be used for sealing fistulas. However, the risk of stent migration is higher than that for uncovered stents because of the low anchoring power. In contrast, uncovered stents have a lower risk for stent migration; however, they have a higher associated tumor ingrowth rate. To overcome this limitation, a partially covered stent that consists of an uncovered segment at both ends of the stent was developed. Two meta-analyses that compared between covered and uncovered stents have shown that covered stents are associated with a higher risk of migration than uncovered stents but have a lower tumor ingrowth. However, there was no significant difference in the technical and clinical success and complication rates between the stent groups. ${ }^{22,23}$ Appropriate stent selection for patients with consideration of clinical situations is very important; however, currently, there is no established approach for selecting the type of stent. To date, the efficacy and safety have been reported to be similar for covered and uncovered stents. Therefore, an endoscopist must be aware of the unique features of each stent type and select the appropriate stent based on patient conditions.

\section{STENT INDICATION}

Two main indications for malignant colonic obstruction are applied in clinical practice: the first approach is palliative stenting for patients with malignant colonic obstruction who are not able to undergo curative surgery and the second approach is preoperative stenting for bowel decompression until the condition is suitable for elective surgical resection, which is also called colonic stenting for BTS. Although a colonic stent can be inserted in any part of the colon, many studies on colonic stenting have focused on left-sided colonic obstruction. This is probably attributable to the relatively lesser severity of right-sided colonic obstruction than of left-sided colonic obstruction. Some retrospective studies have reported successful insertions for proximal colonic obstruction ${ }^{11,24-27}$; however, conflicting results have also been reported. ${ }^{17,28-31}$ Recently, a retrospective multicenter trial reported on the advantages of colonic stents for right-sided colonic obstruction. Sixty-nine patients with a colonic stent and 36 patients who had undergone surgery were included. The technical and clinical success rates of surgery were higher than those of colonic stenting ( $100 \%$ vs. $89.8 \%, p=0.09$ and $100 \%$ vs. $78 \%, p<0.001$ ). However, the surgery group had longer hospital stays ( 8 days vs. 4 days, $p<0.01)$ and higher early adverse event rates $(30.5 \%$ vs. $7.5 \%, p=0.003$ ), while the stenting group had higher rates of late complications, although this result was not statistically significant. ${ }^{32}$ In general, emergency surgery without bowel preparation is considered for patients with right-sided colonic obstruction. However, it should be noted that palliative stenting can be considered as an alternative to surgery for patients who are not able to undergo surgery.

A perforated colon is the only absolute contraindication for colonic stenting. Lower rectal stenting $(\leq 5 \mathrm{~cm}$ from the anal verge) was also considered as a contraindication because of complications, including anal pain, tenesmus, and incontinence. ${ }^{33,34}$ However, a recent Korean retrospective study showed that lower rectal stenting can be an alternative treatment option for obstructive decompression in patients who are not able to undergo surgery. Patients with malignant rectal obstruction underwent lower rectal stenting for palliation or BTS, and the technical and clinical success rates were $87.1 \%$ (34/39) and 69.2\% (27/39), respectively. Only 4 of 27 patients with clinical success reported anal pain, and the pain was well controlled with analgesics. ${ }^{35}$ However, there were several limitations to the study, including the retrospective design and selection bias, because patients with lower rectal obstruction were more likely to undergo palliative surgery rather than stenting. Malignant colonic obstruction can also be caused by extracolonic tumors. However, clinical outcomes of colonic stenting in patients with extracolonic tumors were less favorable than those of colonic stenting in patients with colorectal cancer. ${ }^{36}$ Therefore, stent placement for colonic obstruction by extracolonic tumors should be considered in selective patients who are not suitable for surgery. The indications, clinical advantages, and contraindications of colonic stenting are summarized in Table 1.

\section{Colonic stenting for palliation}

Palliative colonic stenting for malignant obstruction has been demonstrated to be successful enough to be accepted as a first-line treatment. Two recently published meta-analyses that included randomized and non-randomized studies compared the efficacy and safety of colonic stents and emergency surgery in the palliative setting. ${ }^{37,38}$ Clinical relief of obstruction in the surgery group was significantly higher than that of the stent group (99.8\% vs. $93.1 \%, p<0.001)$. However, a meta-analysis showed that the 30-day mortality was lower in the stenting group than in the surgery group $(4.2 \%$ vs. $10.5 \%$, $p=0.01) .{ }^{38}$ In addition, colonic stenting was associated with a shorter hospital stay (9.6 days vs. 18.8 days), lower intensive care unit admission rate ( $0.8 \%$ vs. $18.0 \%)$, and lower stoma formation rate $(12.7 \%$ vs. $54.0 \%){ }^{38}$ The overall complication rates were not significantly different between the groups. The surgery group showed a higher early complication rate, whereas the stenting group had more frequent late compli- 
cations; these findings are consistent with recently published data. ${ }^{32,38}$ Reported colonic stenting-related complications include perforation (12.7\%), stent migration (9.2\%), and stent obstruction (18.3\%). ${ }^{38}$ Another advantage of colonic stenting over surgery for treating malignant obstruction is an earlier start of palliative chemotherapy (15.5 days vs. 33.4 days). ${ }^{38}$ For patients with colonic stents, palliative chemotherapy is associated with better survival rates. ${ }^{36,39}$ Considering these findings, colonic stenting for malignant obstruction can be the preferred treatment option in palliative settings. However, when palliative colonic stenting and bevacizumab treatment are considered for patients with malignant obstructions, careful approaches are needed because of a high risk of stenting-related perforation. Several retrospective studies reported a high risk of stenting-related perforation in patients receiving palliative chemotherapy, especially bevacizumab treatment. ${ }^{17,19,40}$ A meta-analysis that assessed risk factors for stenting-related colonic perforation was recently published. Bevacizumab-based chemotherapy increased the risk of stenting-related perforation (12.5\%) compared with chemotherapy without bevacizumab $(7.0 \%)$ or no chemotherapy $(9.0 \%) .^{41}$ Based on these findings, colonic stenting is not recommended as a firstline treatment option in patients being treated with, or who are candidates for, palliative bevacizumab-based chemotherapy. Another concern related to colonic stenting in patients receiving palliative chemotherapy is that stent migration is associated with tumor shrinkage. ${ }^{42-44}$ Despite these concerns, in patients with malignant obstruction, palliative colonic stenting should be considered as a first-line treatment because of the associated lower short-term mortality rate and earlier start of chemotherapy.

\section{Colonic stenting for bridge to surgery}

Although colonic stents can be inserted in any part of the colon, many studies on colonic stenting have investigated patients with left-sided colonic obstruction. Similarly, most studies on colonic stenting for elective surgery have focused on left-sided malignant obstruction. Symptomatic left-sided malignant colonic obstruction requires bowel decompression via emergency surgery or colonic stenting. Considering the relatively high mortality and morbidity of emergency surgery, colonic stenting appears to be the preferred treatment option for malignant colonic obstruction in patients with potentially curable and resectable cases. ${ }^{45,46}$ Moreover, colonic stenting as a BTS can provide time for staging work-up and optimizing the patients' condition for elective surgery. However, recent studies have not shown a clear superiority of colonic stenting for BTS over emergency surgery. Nine systematic reviews with meta-analyses ${ }^{47-55}$ and eight randomized controlled tri$\mathrm{als}^{56-63}$ that compared the efficacy and safety of colonic stenting for BTS with those of emergency surgery have been published in the last decade. Interestingly, three of the randomized controlled trials were closed prematurely because of contrasting reasons and one was discontinued owing to a high incidence of anastomosis site leakage in the emergency surgery group ${ }^{60}$ and the other two owing to higher rates of adverse events, such as stenting-related perforation in the stenting group. ${ }^{61,62}$

The most recently published systematic review with meta-analysis compared the efficacy and safety of colonic stenting for BTS with those of emergency surgery, and only randomized controlled trials were considered for inclusion. ${ }^{47} \mathrm{~A}$ total of 497 patients were included (251 in the stenting group vs. 246 in the emergency surgery group). The overall shortterm mortality (within 60 days after surgery) was not significantly different between these groups ( $9.6 \%$ in the stenting group vs. $9.9 \%$ in the emergency surgery group). ${ }^{47}$ However, the overall short-term morbidity was significantly lower in the stenting group than in the emergency surgery group $(33.9 \%$

Table 1. Indication of Colonic Stent and Each Clinical Advantages over Surgery

\begin{tabular}{lll}
\hline Indication & \multicolumn{1}{c}{ Advantage } & \multicolumn{1}{c}{ Disadvantage } \\
\hline Colonic stent for palliation & $\begin{array}{l}\text { Lower short term motality } \\
\text { Shorter hospital day } \\
\text { Lower stoma rate } \\
\text { Earlier start of chemotherapy }\end{array}$ & $\begin{array}{l}\text { High stent related perforation risk } \\
\text { (especially Bevacizumab) }\end{array}$ \\
Colonic stent for bridge to surgery & $\begin{array}{l}\text { Lower short term morbidity } \\
\text { Lower stoma rate }\end{array}$ & Concern about oncological outcomes \\
& High primary anastomosis rate & Possibility of surgical failure \\
Contraindication & \\
Perforated colon (absolute) & \\
Lower rectal stenting $(<5 \mathrm{~cm}$ from anal verge, relative) & \\
Extrinsic compression by extracolonic tumors (relative) & \\
\hline
\end{tabular}


vs. $51.2 \%, p=0.023){ }^{47}$ In addition to morbidity, the meta-analysis showed that the stenting group had a lower temporary stoma rate (33.9\% vs. $51.4 \%, p<0.001)$, lower permanent stoma rate $(22.2 \%$ vs. $35.2 \%, p=0.003)$, and higher primary anastomosis rate $(70.0 \%$ vs. $54.1 \%, p=0.043){ }^{47}$ These findings are consistent with those of a previous meta-analysis. ${ }^{48}$ Based on these results, colonic stenting for BTS in patients with malignant obstruction, which is potentially curable and resectable, may be more advantageous than emergency surgery in regard to short-term outcomes. However, currently, there are conflicting data on colonic stenting for BTS. One study reported that the use of multiple colonic stents for BTS is associated with surgical failure. ${ }^{61}$ Long-term outcomes, especially oncological outcomes of colonic stenting for BTS, are another major concern. One comparative prospective study showed that the local recurrence rate was significantly higher in the stenting group than in the primary surgery group ${ }^{64}$ In the Dutch Stent-In 2 trial, colonic stenting for BTS and stenting-related perforation were associated with a higher overall recurrence rate. ${ }^{65}$ Recently, a systematic review with meta-analysis that included 21 comparative studies was published; this study evaluated the oncological safety of colonic stenting for BTS in patients with malignant left-sided obstruction. ${ }^{66} \mathrm{~A}$ total of 1,919 patients were included (938 in the stenting group vs. 981 in the emergency surgery group). There were no significant differences in the 3- and 5-year overall survival rates between the two groups $(69.7 \%$ vs. $67.9 \%$ and $63.5 \%$ vs. $57.9 \%$, respectively). ${ }^{66}$ The disease-free survival and overall and local recurrence rates were not significantly different. ${ }^{66}$ The permanent stoma rate was significantly lower in the stenting group than in the emergency surgery group ( $14.7 \%$ vs. $26.5 \%$; odds ratio [OR], 0.49; 95\% confidence interval [CI], 0.32-0.74). ${ }^{66}$ These results may indicate that colonic stenting for BTS in patients with malignant left-sided obstruction yields favorable oncological safety profiles and lower permanent stoma rates than does emergency surgery. However, careful interpretation of these results is necessary because of conflicting data on survival in the stenting groups based on subgroup analyses that included only randomized controlled trials (all four trials). In the subgroup analysis, the stenting group had worse survival outcomes (OR, 1.18; 95\% CI, 0.66-2.11), although this result was not statistically significant. The meta-analysis was mainly based on non-randomized studies; therefore, there is still a need for more randomized studies to assess the oncological outcomes of colonic stenting for BTS clearly in patients with malignant obstructions. However, despite the current uncertainty on oncological outcomes, colonic stenting for BTS is advantageous compared with emergency surgery because of the lower short-term morbidity and temporary or permanent stoma rates and higher primary anastomosis rates. ${ }^{47}$
In addition, colonic stenting for BTS can facilitate elective laparoscopic resection. A population-based study showed that the postoperative mortality in laparoscopic colorectal cancer surgery is lower than that in open surgery. ${ }^{67}$ Considering these advantages, colonic stenting for BTS could be performed as an alternative treatment option for patients with high surgical risks, such as those with advanced age, multiple co-morbidities, and high American Society of Anesthesiologists (ASA) classification. ${ }^{68-73}$ This is consistent with the current ESGE guidelines for malignant colonic obstruction. The guidelines suggest that colonic stenting for BTS could be an alternative treatment in patients who have an increased risk of postoperative mortality, especially those with an ASA classification of $\geq$ III or an age of $>70$ years.

\section{COMPLICATIONS}

Colonic stenting for malignant obstruction is a relatively low-risk procedure with a mortality rate of $<4 \%{ }^{28,38,74}$ However, it is associated with various complications, such as perforation, migration, re-obstruction, pain, and bleeding. Colonic stenting-related complications are usually divided into early ( $\leq 30$ days) and late ( $>30$ days) complications. Perforation is the most serious colonic stenting-related complication, with a mortality rate of $16 \%$, although the overall risk is relatively low. ${ }^{75,76}$ Guidewire or catheter misplacements are frequently associated with early perforation, while stent quality can be a cause of late perforation. ${ }^{21}$ As mentioned above, colonic stenting in patients who will receive bevacizumab treatment should be avoided as much as possible because of the high risk of perforation. ${ }^{17,19,40}$ When perforation is documented, emergency surgery is usually required. However, some patients with microperforation can be treated with antibiotics and bowel rest. ${ }^{77}$ Stent migration can also be an early or a late complication. Use of covered stents, small stent diameter of $17-21 \mathrm{~cm}(<24 \mathrm{~cm})$, too short in length to obstructive lesion, and chemotherapy-induced tumor shrinkage are factors that might affect stent migration. ${ }^{17-23,42-44}$ Stent re-obstruction by

Table 2. Complications of Colonic Stent and Proper Management

\begin{tabular}{ll}
\hline Complications & \multicolumn{1}{c}{ Management } \\
\hline Major & \\
Perforation & Emergency surgery except microperforation \\
Migration & Replacement of SEMS \\
Re-obstruction & Additional SEMS \\
Minor & \\
Pain, bleeding & Conservative \\
\hline
\end{tabular}

SEMS, self-expandable metal stent. 
tumor in- or overgrowth is a late complication of colonic stenting, especially in the palliative setting. Previous studies have reported a median stent patency duration of 106 days and that the use of uncovered stents is a risk factor for tumor ingrowth. ${ }^{22,76}$ Stent migration and re-obstruction can be managed with stent replacement and additional stenting. ${ }^{39,78,79}$ Post-stenting bleeding and pain are minor complications and can be managed conservatively in the majority of patients. ${ }^{11}$ The complications of colonic stenting and their proper management are summarized in Table 2.

\section{CONCLUSIONS}

Endoscopic management of malignant colonic obstruction using SEMSs can be performed as a palliative therapy or BTS. Since palliative colonic stenting for malignant obstruction is associated with lower morbidity and mortality rates and an earlier implementation of palliative chemotherapy, it may be accepted as a first-line treatment. Colonic stenting for BTS also yielded better outcomes than emergency surgery, although using SEMSs for patients with resectable malignant colonic obstructions, especially in cases with uncertain oncological outcomes, remains controversial. Colonic stenting for BTS can be an alternative treatment option for patients with high surgical risks, such as those with advanced age, multiple co-morbidities, and high ASA classification.

\section{Conflicts of Interest}

The authors have no financial conflicts of interest.

\section{ORCID}

Seung Young Seo: https://orcid.org/0000-0003-2018-0013

\section{REFERENCES}

1. Billingsley KG, Morris AM, Dominitz JA, et al. Surgeon and hospital characteristics as predictors of major adverse outcomes following colon cancer surgery: understanding the volume-outcome relationship. Arch Surg 2007;142:23-31; discussion 32.

2. Deans GT, Krukowski ZH, Irwin ST. Malignant obstruction of the left colon. Br J Surg 1994;81:1270-1276.

3. Ascanelli S, Navarra G, Tonini G, et al. Early and late outcome after surgery for colorectal cancer: elective versus emergency surgery. Tumori 2003;89:36-41.

4. Serpell JW, McDermott FT, Katrivessis H, Hughes ES. Obstructing carcinomas of the colon. Br J Surg 1989;76:965-969.

5. Jemal A, Bray F, Center MM, Ferlay J, Ward E, Forman D. Global cancer statistics. CA Cancer J Clin 2011;61:69-90.

6. Gordon PH. Malignant neoplasms of the colon. In: Gordon PH, Nivatvongs S, eds. Principles and practice of surgery for the colon, rectum, and anus. St. Louis (MO): Quality Medical Publishing; 1999. p. 575-717.

7. Anderson JH, Hole D, McArdle CS. Elective versus emergency surgery for patients with colorectal cancer. Br J Surg 1992;79:706-709.

8. Copeland GP, Sagar P, Brennan J, et al. Risk-adjusted analysis of surgeon performance: a 1-year study. Br J Surg 1995;82:408-411.

9. Longo WE, Virgo KS, Johnson FE, et al. Outcome after proctectomy for rectal cancer in Department of Veterans Affairs Hospitals: a report from the National Surgical Quality Improvement Program. Ann Surg 1998;228:64-70.

10. Chun YJ, Yoon NR, Park JM, et al. Prospective assessment of risk of bacteremia following colorectal stent placement. Dig Dis Sci 2012;57:10451049.

11. Geraghty J, Sarkar S, Cox T, et al. Management of large bowel obstruction with self-expanding metal stents. A multicentre retrospective study of factors determining outcome. Colorectal Dis 2014;16:476-483.

12. Kim JW, Jeong JB, Lee KL, et al. Comparison of clinical outcomes between endoscopic and radiologic placement of self-expandable metal stent in patients with malignant colorectal obstruction. Korean J Gastroenterol 2013;61:22-29.

13. Sebastian S, Johnston S, Geoghegan T, Torreggiani W, Buckley M. Pooled analysis of the efficacy and safety of self-expanding metal stenting in malignant colorectal obstruction. Am J Gastroenterol 2004;99:2051-2057.

14. de Gregorio MA, Laborda A, Tejero E, et al. Ten-year retrospective study of treatment of malignant colonic obstructions with self-expandable stents. J Vasc Interv Radiol 2011;22:870-878.

15. Williams D, Law R, Pullyblank AM. Colorectal stenting in malignant large bowel obstruction: the learning curve. Int J Surg Oncol 2011;2011:917848.

16. Lee JH, Yoon JY, Park SJ, et al. The learning curve for colorectal stent insertion for the treatment of malignant colorectal obstruction. Gut Liver 2012;6:328-333.

17. Small AJ, Coelho-Prabhu N, Baron TH. Endoscopic placement of self-expandable metal stents for malignant colonic obstruction: long-term outcomes and complication factors. Gastrointest Endosc 2010;71:560-572.

18. Kim BC, Han KS, Hong CW, et al. Clinical outcomes of palliative self-expanding metallic stents in patients with malignant colorectal obstruction. J Dig Dis 2012;13:258-266.

19. Manes G, de Bellis M, Fuccio L, et al. Endoscopic palliation in patients with incurable malignant colorectal obstruction by means of self-expanding metal stent: analysis of results and predictors of outcomes in a large multicenter series. Arch Surg 2011;146:1157-1162.

20. Im JP, Kim SG, Kang HW, Kim JS, Jung HC, Song IS. Clinical outcomes and patency of self-expanding metal stents in patients with malignant colorectal obstruction: a prospective single center study. Int J Colorectal Dis 2008;23:789-794.

21. Baron TH, Wong Kee Song LM, Repici A. Role of self-expandable stents for patients with colon cancer (with videos). Gastrointest Endosc 2012;75:653-662.

22. Zhang Y, Shi J, Shi B, Song CY, Xie WF, Chen YX. Comparison of efficacy between uncovered and covered self-expanding metallic stents in malignant large bowel obstruction: a systematic review and meta-analysis. Colorectal Dis 2012;14:e367-e374.

23. Yang Z, Wu Q, Wang F, Ye X, Qi X, Fan D. A systematic review and meta-analysis of randomized trials and prospective studies comparing covered and bare self-expandable metal stents for the treatment of malignant obstruction in the digestive tract. Int J Med Sci 2013;10:825-835.

24. Yoon JY, Jung YS, Hong SP, Kim TI, Kim WH, Cheon JH. Clinical outcomes and risk factors for technical and clinical failures of self-expandable metal stent insertion for malignant colorectal obstruction. Gastrointest Endosc 2011;74:858-868.

25. Cho YK, Kim SW, Lee BI, et al. Clinical outcome of self-expandable metal stent placement in the management of malignant proximal colon obstruction. Gut Liver 2011;5:165-170.

26. Yao LQ, Zhong YS, Xu MD, Xu JM, Zhou PH, Cai XL. Self-expanding metallic stents drainage for acute proximal colon obstruction. World J 
Gastroenterol 2011;17:3342-3346.

27. Repici A, Adler DG, Gibbs CM, Malesci A, Preatoni P, Baron TH. Stenting of the proximal colon in patients with malignant large bowel obstruction: techniques and outcomes. Gastrointest Endosc 2007;66:940944.

28. Meisner S, González-Huix F, Vandervoort JG, et al. Self-expandable metal stents for relieving malignant colorectal obstruction: short-term safety and efficacy within 30 days of stent procedure in 447 patients. Gastrointest Endosc 2011;74:876-884.

29. Selinger CP, Ramesh J, Martin DF. Long-term success of colonic stent insertion is influenced by indication but not by length of stent or site of obstruction. Int J Colorectal Dis 2011;26:215-218.

30. Kim JY, Kim SG, Im JP, Kim JS, Jung HC. Comparison of treatment outcomes of endoscopic stenting for colonic and extracolonic malignant obstruction. Surg Endosc 2013;27:272-277.

31. Dronamraju SS, Ramamurthy S, Kelly SB, Hayat M. Role of self-expanding metallic stents in the management of malignant obstruction of the proximal colon. Dis Colon Rectum 2009;52:1657-1661.

32. Siddiqui A, Cosgrove N, Yan LH, et al. Long-term outcomes of palliative colonic stenting versus emergency surgery for acute proximal malignant colonic obstruction: a multicenter trial. Endosc Int Open 2017;5:E232-E238.

33. ASGE Standards of Practice Committee, Harrison ME, Anderson MA, et al. The role of endoscopy in the management of patients with known and suspected colonic obstruction and pseudo-obstruction. Gastrointest Endosc 2010;71:669-679.

34. Hünerbein M, Krause M, Moesta KT, Rau B, Schlag PM. Palliation of malignant rectal obstruction with self-expanding metal stents. Surgery 2005;137:42-47.

35. Lee HJ, Hong SP, Cheon JH, Kim TI, Kim WH, Park SJ. Clinical outcomes of self-expandable metal stents for malignant rectal obstruction. Dis Colon Rectum 2018;61:43-50.

36. Luigiano C, Ferrara F, Fabbri C, et al. Through-the-scope large diameter self-expanding metal stent placement as a safe and effective technique for palliation of malignant colorectal obstruction: a single center experience with a long-term follow-up. Scand J Gastroenterol 2011;46:591-596.

37. Liang TW, Sun Y, Wei YC, Yang DX. Palliative treatment of malignant colorectal obstruction caused by advanced malignancy: a self-expanding metallic stent or surgery? A system review and meta-analysis. Surg Today 2014;44:22-33.

38. Zhao XD, Cai BB, Cao RS, Shi RH. Palliative treatment for incurable malignant colorectal obstructions: a meta-analysis. World J Gastroenterol 2013;19:5565-5574.

39. Lee HJ, Hong SP, Cheon JH, et al. Long-term outcome of palliative therapy for malignant colorectal obstruction in patients with unresectable metastatic colorectal cancers: endoscopic stenting versus surgery. Gastrointest Endosc 2011;73:535-542.

40. Cennamo V, Fuccio L, Mutri V, et al. Does stent placement for advanced colon cancer increase the risk of perforation during bevacizumab-based therapy? Clin Gastroenterol Hepatol 2009;7:1174-1176.

41. van Halsema EE, van Hooft JE, Small AJ, et al. Perforation in colorectal stenting: a meta-analysis and a search for risk factors. Gastrointest Endosc 2014;79: 970-82.e7; quiz 983.e2, 983.e5.

42. Kim JH, Song HY, Li YD, et al. Dual-design expandable colorectal stent for malignant colorectal obstruction: comparison of flared ends and bent ends. AJR Am J Roentgenol 2009;193:248-254.

43. Canena JM, Liberato M, Marques I, et al. Sustained relief of obstructive symptoms for the remaining life of patients following placement of an expandable metal stent for malignant colorectal obstruction. Rev Esp Enferm Dig 2012;104:418-425.

44. Fernández-Esparrach G, Bordas JM, Giráldez MD, et al. Severe complications limit long-term clinical success of self-expanding metal stents in patients with obstructive colorectal cancer. Am J Gastroenterol 2010;105:1087-1093.

45. McLoughlin MT, Byrne MF. Endoscopic stenting: where are we now and where can we go? World J Gastroenterol 2008;14:3798-3803.

46. Law WL, Choi HK, Chu KW. Comparison of stenting with emergency surgery as palliative treatment for obstructing primary left-sided colorectal cancer. Br J Surg 2003;90:1429-1433.

47. Arezzo A, Passera R, Lo Secco G, et al. Stent as bridge to surgery for left-sided malignant colonic obstruction reduces adverse events and stoma rate compared with emergency surgery: results of a systematic review and meta-analysis of randomized controlled trials. Gastrointest Endosc 2017;86:416-426.

48. Huang X, Lv B, Zhang S, Meng L. Preoperative colonic stents versus emergency surgery for acute left-sided malignant colonic obstruction: a meta-analysis. J Gastrointest Surg 2014;18:584-591.

49. Cennamo V, Luigiano C, Coccolini F, et al. Meta-analysis of randomized trials comparing endoscopic stenting and surgical decompression for colorectal cancer obstruction. Int J Colorectal Dis 2013;28:855-863.

50. Cirocchi R, Farinella E, Trastulli S, et al. Safety and efficacy of endoscopic colonic stenting as a bridge to surgery in the management of intestinal obstruction due to left colon and rectal cancer: a systematic review and meta-analysis. Surg Oncol 2013;22:14-21.

51. De Ceglie A, Filiberti R, Baron TH, Ceppi M, Conio M. A meta-analysis of endoscopic stenting as bridge to surgery versus emergency surgery for left-sided colorectal cancer obstruction. Crit Rev Oncol Hematol 2013;88:387-403.

52. Tan CJ, Dasari BV, Gardiner K. Systematic review and meta-analysis of randomized clinical trials of self-expanding metallic stents as a bridge to surgery versus emergency surgery for malignant left-sided large bowel obstruction. Br J Surg 2012;99:469-476.

53. Ye GY, Cui Z, Chen L, Zhong M. Colonic stenting vs emergent surgery for acute left-sided malignant colonic obstruction: a systematic review and meta-analysis. World J Gastroenterol 2012;18:5608-5615.

54. Zhang Y, Shi J, Shi B, Song CY, Xie WF, Chen YX. Self-expanding metallic stent as a bridge to surgery versus emergency surgery for obstructive colorectal cancer: a meta-analysis. Surg Endosc 2012;26:110-119.

55. Sagar J. Colorectal stents for the management of malignant colonic obstructions. Cochrane Database Syst Rev 2011;(11):CD007378.

56. Arezzo A, Balague C, Targarona E, et al. Colonic stenting as a bridge to surgery versus emergency surgery for malignant colonic obstruction: results of a multicentre randomised controlled trial (ESCO trial). Surg Endosc 2017;31:3297-3305.

57. Ghazal AH, El-Shazly WG, Bessa SS, El-Riwini MT, Hussein AM. Colonic endolumenal stenting devices and elective surgery versus emergency subtotal/total colectomy in the management of malignant obstructed left colon carcinoma. J Gastrointest Surg 2013;17:1123-1129.

58. Tung KL, Cheung HY, Ng LW, Chung CC, Li MK. Endo-laparoscopic approach versus conventional open surgery in the treatment of obstructing left-sided colon cancer: long-term follow-up of a randomized trial. Asian J Endosc Surg 2013;6:78-81.

59. Ho KS, Quah HM, Lim JF, Tang CL, Eu KW. Endoscopic stenting and elective surgery versus emergency surgery for left-sided malignant colonic obstruction: a prospective randomized trial. Int J Colorectal Dis 2012;27:355-362.

60. Alcántara M, Serra-Aracil X, Falcó J, Mora L, Bombardó J, Navarro S. Prospective, controlled, randomized study of intraoperative colonic lavage versus stent placement in obstructive left-sided colonic cancer. World J Surg 2011;35:1904-1910.

61. Pirlet IA, Slim K, Kwiatkowski F, Michot F, Millat BL. Emergency preoperative stenting versus surgery for acute left-sided malignant colonic obstruction: a multicenter randomized controlled trial. Surg Endosc 2011;25:1814-1821.

62. van Hooft JE, Bemelman WA, Oldenburg B, et al. Colonic stenting versus emergency surgery for acute left-sided malignant colonic obstruction: a multicentre randomised trial. Lancet Oncol 2011;12:344-352.

63. Cheung HY, Chung CC, Tsang WW, Wong JC, Yau KK, Li MK. Endolaparoscopic approach vs conventional open surgery in the treatment of obstructing left-sided colon cancer: a randomized controlled trial. 
Arch Surg 2009;144:1127-1132.

64. Gorissen KJ, Tuynman JB, Fryer E, et al. Local recurrence after stenting for obstructing left-sided colonic cancer. Br J Surg 2013;100:1805-1809.

65. Sloothaak DA, van der Berg MW, Dijkgraaf MG, et al. Recurrences after endoscopic stenting as treatment for acute malignant colonic obstruction in the Dutch stent-in 2 trial. In: 21st United European Gastroenterology Week; 2013 Oct 12-16; Berlin, Germany. Vienna: United European Gastroenterology; 2013. p. A132.

66. Amelung FJ, Burghgraef TA, Tanis PJ, et al. Critical appraisal of oncological safety of stent as bridge to surgery in left-sided obstructing colon cancer; a systematic review and meta-analysis. Crit Rev Oncol Hematol 2018;131:66-75.

67. Gietelink L, Wouters MW, Bemelman WA, Dekker JW, Tollenaar RA, Tanis PJ. Reduced 30-day mortality after laparoscopic colorectal cancer surgery: a population based study from the Dutch Surgical Colorectal Audit (DSCA). Ann Surg 2016;264:135-140.

68. Jullumstrø E, Wibe A, Lydersen S, Edna TH. Colon cancer incidence, presentation, treatment and outcomes over 25 years. Colorectal Dis 2011;13:512-518.

69. Biondo S, Parés D, Frago R, et al. Large bowel obstruction: predictive factors for postoperative mortality. Dis Colon Rectum 2004;47:18891897.

70. Tekkis PP, Kinsman R, Thompson MR, Stamatakis JD. The Association of Coloproctology of Great Britain and Ireland study of large bowel obstruction caused by colorectal cancer. Ann Surg 2004;240:76-81.

71. Tan KK, Sim R. Surgery for obstructed colorectal malignancy in an
Asian population: predictors of morbidity and comparison between leftand right-sided cancers. J Gastrointest Surg 2010;14:295-302.

72. Iversen LH. Aspects of survival from colorectal cancer in Denmark. Dan Med J 2012;59:B4428.

73. Symeonidis D, Christodoulidis G, Koukoulis G, Spyridakis M, Tepetes K. Colorectal cancer surgery in the elderly: limitations and drawbacks. Tech Coloproctol 2011;15 Suppl 1:S47-S50.

74. Abbott S, Eglinton TW, Ma Y, Stevenson C, Robertson GM, Frizelle FA. Predictors of outcome in palliative colonic stent placement for malignant obstruction. Br J Surg 2014;101:121-126.

75. Datye A, Hersh J. Colonic perforation after stent placement for malignant colorectal obstruction--causes and contributing factors. Minim Invasive Ther Allied Technol 2011;20:133-140.

76. Watt AM, Faragher IG, Griffin TT, Rieger NA, Maddern GJ. Self-expanding metallic stents for relieving malignant colorectal obstruction: a systematic review. Ann Surg 2007;246:24-30.

77. Lopera JE, De Gregorio MA. Fluoroscopic management of complications after colorectal stent placement. Gut Liver 2010;4 Suppl 1:S9-S18.

78. Yoon JY, Park SJ, Hong SP, Kim TI, Kim WH, Cheon JH. Outcomes of secondary self-expandable metal stents versus surgery after delayed initial palliative stent failure in malignant colorectal obstruction. Digestion 2013;88:46-55.

79. Yoon JY, Jung YS, Hong SP, Kim TI, Kim WH, Cheon JH. Outcomes of secondary stent-in-stent self-expandable metal stent insertion for malignant colorectal obstruction. Gastrointest Endosc 2011;74:625-633. 